\title{
Estructura y daños en las comunidades de octocorales (Octocorallia: Alcyonacea) de la Reserva Ecológica Siboney-Juticí, Santiago de Cuba, Cuba
}

\author{
Yunier Olivera Espinosa ${ }^{1}$, Leslie Hernández-Fernández ${ }^{1} \&$ Abdiel Jover Capote ${ }^{2}$ \\ 1. Centro de Investigaciones de Ecosistemas Costeros (CIEC), Ministerio de Ciencia Tecnología y Medio Ambiente, \\ Cayo Coco, Jardines del Rey, Ciego de Ávila, Cuba; yunier@ciec.fica.inf.cu, leslie@ciec.fica.inf.cu \\ 2. Departamento de Biología, Facultad de Ciencias Naturales, Universidad de Oriente, Patricio Lumumba s/n, Santiago \\ de Cuba, Cuba; abdiel@cnt.uo.edu.cu
}

Received 20-I-2010. C Corrected 15-VI-2010. Accepted 19-VII-2010.

\begin{abstract}
Structure and injuries of octocoral communities (Octocorallia: Alcyonacea) of Ecological Reserve Siboney-Juticí, Santiago de Cuba, Cuba. In the spur-and-groove reefs of the Ecological Reserve Siboney-Juticí (Southeast Cuba) octocorals are one of the predominant components of the sessile fauna. Main objectives of the present paper are characterizing the composition, structure and conservation status of the octocoral communities and assessing on the prevailing environmental conditions in the study area. For data collection, six sampling sites were located every $2-3 \mathrm{~km}$ ranging from $12 \mathrm{~m}$ to $17 \mathrm{~m}$ depth along the spur-and-groove reefs. In each site, 22-26 $1 \mathrm{~m}^{2}$ quadrants were zigzag arranged every $2 \mathrm{~m}$. The density of colonies was determined per site. The severity degree and predictability of environmental conditions were inferred by using the Heterogeneity and Equitability indexes, respectively. Hydrodynamic stress and the Comparative Pollution Index were also assessed. Current affectations of the octocoral communities were also determined and classified into four main categories: mechanical damage, diseases, predation and invertebrate and macroalgae overgrowth. As a result, 25 species represented by 752 colonies were recorded and Eunicea flexuosa and Gorgonia ventalina were the most abundant. The occurrence of Eunicea succinea forma succinea constituted the first report for Eastern Cuba. Site densities ranged from $3.58 \pm 1.84$ to $7.58 \pm 2.16$ colonies $/ \mathrm{m}^{2}$ and considered from moderate to low. The biggest densities were reported at both sides of the San Juan River mouth. Despite of the composition and structure of the octocoral communities, low to high hydrodynamic stress and low and moderate levels of contamination were inferred, it is likely that these indexes could have been overestimated due to the sensitivity of the indicator species to other factors such as sedimentation. The environmental conditions were mostly favorable and stable. In general, the number of dead colonies was low and mostly caused by the detachment of the substrate and overgrowth of Millepora alcicornis. Injuries were mainly brought about by mechanical damage, followed by coenenchyme loss, predation by Cyphoma gibbosum and macroalgae overgrowth. On the other hand, negative effects by Hermodice carunculata predation, bleaching, Red Band and Black Band Disease were not observed. Among sampled species, G. ventalina colonies were the most severely damaged within the community. As a whole, octocoral communities of spur-and-groove reefs in the Ecological Reserve Siboney-Juticí showed a good conservation status. Rev. Biol. Trop. 58 (4): 1211-1221. Epub 2010 December 01.
\end{abstract}

Key words: octocorals, spur-and-groove reefs, composition, structure, injuries, Cuba.

Los octocorales se ubican entre los grupos de invertebrados marinos predominantes en número y biomasa de la fauna sésil de los arrecifes coralinos en el Caribe, por lo que desempeñan un importante papel como hábitat y refugio de otros organismos, debido a que aumentan la complejidad estructural del arrecife. También son componentes de la red alimentaria arrecifal y constituyen uno de los grupos focales en la detección de perturbaciones en el ambiente marino, con dependencia de la abundancia o escasez de determinadas 
especies (Alcolado 1981, García-Parrado \& Alcolado 1996).

Los estudios sobre las comunidades de octocorales realizados a inicios del siglo pasado en el Caribe, fueron escasos y no se hicieron sistemáticamente hasta la segunda mitad del mismo. Entre ellos se destacaron los realizados por Guitart-Manday (1959), Bayer (1961), Kinzie (1973), Goldberg (1973) y Preston \& Preston (1975), enfocados en la taxonomía y estructura de las comunidades. En Cuba, la mayoría de las investigaciones se han realizado en las regiones central y occidental, como las de Herrera-Moreno \& Alcolado (1983, 1986), Herrera-Moreno (1991), González-Díaz et al. (2003), González-Ontivero et al. (2007) y Hernández-Fernández \& Alcolado (2007), entre otros. Específicamente, en la costa sur oriental del territorio nacional solo se han efectuado dos investigaciones con este fin (Alcolado 1981, Chiappone et al. 2001), pero no han incluido un estudio exhaustivo de dichas comunidades en la Reserva Ecológica Siboney-Juticí.

Para el manejo y conservación de las áreas marinas protegidas es necesario conocer los componentes, estructura y funcionamiento de sus comunidades, así como determinar el estado de conservación de éstas y los principales factores abióticos que rigen su ecología. Con este propósito y el de inferir el grado de severidad y constancia ambiental, se estudió la comunidad de octocorales en la Reserva Ecológica Siboney-Juticí, donde estos organismos están entre los elementos sésiles predominantes.

\section{MATERIALES Y MÉTODOS}

Área de estudio: El trabajo se realizó entre enero y junio de 2009, en la Reserva Ecológica Siboney-Juticí (1956“26”-1958“13” $\mathrm{N}, 75^{\circ} 49^{\prime} 32^{\prime}$ - $75^{\circ} 42^{\prime} 24^{\prime}$ 'W), núcleo de conservación de la Reserva de Biosfera Baconao, situada en la costa suroriental de Cuba, específicamente en el sector costero entre las bahías de Santiago de Cuba y Guantánamo. La terraza marina en esta zona del país es muy estrecha y se caracteriza por presentar fondos de macizos y canales, conocidos en inglés como "spursand-grooves".

Método de muestreo: Se establecieron 6 localidades entre $12 \mathrm{~m}$ y $17 \mathrm{~m}$ de profundidad. Las localidades se denominaron: Juticí, El Mangle, Sardinero este, Sardinero oeste, Aguadores este y Aguadores oeste; las dos últimas a ambos lados de la desembocadura del río San Juan. Como unidad de muestreo se empleó un marco cuadrado de $1 \mathrm{~m}$ de lado (Weinberg 1981). Dentro de cada marco se identificaron las especies presentes y se determinó el número de colonias de cada una. A las colonias que no pudieron ser identificadas in situ se les cortó una porción para su posterior identificación microscópica por medio de espículas, siguiendo los criterios de Bayer (1961).

El marco cuadrado se ubicó en zigzag cada $2 \mathrm{~m}$ sobre los macizos hasta alcanzar entre 22 y 26 unidades de muestreo por cada localidad. La distribución de las unidades de muestreo se realizó de forma tal que cubrieran la mayor parte de la superficie de los macizos teniendo en cuenta el tamaño, por lo general pequeño, de estas estructuras rocoso-coralinas, evitando siempre sus bordes. Para determinar si el tamaño de muestra fue representativo, se elaboraron curvas acumulativas del número de especies contra unidades de muestreo con 999 permutaciones, empleando para ello el software PRIMER 5.

En cada localidad se estimó el porcentaje y la densidad de colonias. Esta última se clasificó teniendo en cuenta los criterios de Alcolado (com. pers. ${ }^{1}$ ): 0-5.0 colonias $/ \mathrm{m}^{2}=$ baja, 5.1-10.0 colonias $/ \mathrm{m}^{2}=$ moderada, 10.1-15.0 colonias/ $\mathrm{m}^{2}=$ alta, $15.1-20.0$ colonias $/ \mathrm{m}^{2}=$ muy alta .

Para conocer la estructura de las comunidades de octocorales de la zona de estudio, se determinaron los índices ecológicos de Riqueza Específica $\left(\mathrm{S}_{\mathrm{obs}}\right)$ según Ludwing \& Reynolds

1. Dr. Pedro M. Alcolado, Investigador Titular de Sistemática y Ecología de corales, Instituto de Oceanología de Cuba. 
(1988), Heterogeneidad (H') de Shannon \& Weaver (1949), usando el logaritmo neperiano y Equitatividad (J') de Pielou (1966). Para inferir el grado de severidad y constancia ambiental para los octocorales en cada localidad, se interpretaron los índices de H'y J', según la modificación del método de Preston \& Preston (1975) realizada por Alcolado (1992) para esponjas y adaptada para octocorales por HernándezFernández \& Alcolado (2007) (Cuadro 1).

A partir de las densidades por especies, se confeccionó una matriz de afinidad utilizándose el Índice de Disimilitud de Bray-Curtis (Bray \& Curtis 1957). En el cálculo numérico posterior se empleó un programa de clasificación jerárquica, aplicándose el UPGMA como estrategia aglomerativa. Para ello se utilizó el software PRIMER 5.

Para inferir el grado de agitación del agua en cada localidad, se determinó el Índice de Tensión Hidrodinámica (ITH) de Alcolado (1981), basado en la suma de los porcentajes de las especies de octocorales resistentes a este tensor: Muricea muricata (Pallas 1766), Eunicea calyculata f. calyculata Bayer 1961, Eunicea tourneforti Milne Edwards \& Haime 1857, Eunicea flexuosa (Lamouroux 1821), Eunicea mammosa Lamouroux 1816, Plexaurella dichotoma (Esper 1791), Gorgonia ventalina Linnaeus 1758, Gorgonia flabellum Linnaeus 1758, Pterogorgia anceps (Pallas 1766), Pterogorgia citrina (Esper 1792) y Pterogorgia guadalupensis Duchassaing \& Michelin 1846. Este se valoró como: $0-20 \%=$ muy bajo, $21-40 \%=$ bajo, $41-60 \%=$ moderado, $61-80 \%=$ alto, $81-100 \%=$ muy alto. Se consideró, además, el Índice Comparativo de Contaminación (ICC) propuesto por Herrera-Moreno (1991), basado en la suma de los porcentajes de las especies consideradas por este autor como resistentes a la contaminación: Eunicea calyculata f. coronata Bayer 1961, E. calyculata f. calyculata, E. flexuosa, E. mammosa, E. tourneforti, Plexaura kuekenthali Moser 1921 y Pseudoplexaura flagellosa (Houtuyn 1772).

Colonias afectadas: Dentro de los marcos se contaron las colonias muertas y afectadas y se determinó el tipo de daño presente. En la categoría de colonias dañadas se incluyeron las colonias rotas por acción mecánica, pérdida de cenénquima, blanqueamiento, daño por depredación de Cyphoma gibbosum (Linnaeus 1758) o Hermodice carunculata (Pallas 1766), por sobrecrecimiento de Millepora alcicornis Linnaeus 1758, u otros invertebrados y macroalgas, así como por la presencia de enfermedades como Banda Negra, Banda Roja, tumores algales y aspergilosis.

\section{RESULTADOS}

Composición, abundancia y densidad: En la zona se contaron e identificaron 752

CUADRO 1

Severidad y pronosticabilidad ambiental inferida para octocorales, según las combinaciones de los índices de Heterogeneidad ( $\left.H^{\prime}\right)$ y de Equitatividad ( $\left.J^{\prime}\right)$

TABLE 1

Environmental severity and predictability inferred for octocorals, according to the combinations of Heterogeneity $\left(H^{\prime}\right)$ and Equitability $\left(J^{\prime}\right)$ indexes

$\begin{array}{clcl}\text { Valores de } \mathrm{H}^{\prime} & \text { Comportamiento ambiental } & \text { Valores de J' } & \text { Estado ambiental } \\ 0-0.99 & \text { muy severo } & <0.7 & \text { no pronosticable } \\ 1.0-1.99 & \text { severo } & 0.7-0.79 & \text { casi constante } \\ 2.0-2.39 & \text { favorable } & >0.79 & \text { constante } \\ >2.39 & \text { muy favorable } & & \end{array}$

Tomado de Hernández-Fernández \& Alcolado (2007). 
colonias de octocorales, pertenecientes a 25 especies, cuyo número por localidad de muestreo varió entre 9 y 16. Las especies más abundantes en el área de estudio fueron: $E$. flexuosa (19\%) y G. ventalina (17\%), seguidas por Plexaura homomalla (Esper 1792) (11\%), Pseudopterogorgia americana (Gmelin 1791) (9\%) y Muriceopsis flavida (Lamarck 1815) (8\%) (Cuadro 2). La especie Eunicea succinea f. succinea Bayer 1961 se consignó como nueva para la zona oriental de Cuba.
La densidad de colonias fluctuó entre $3.58 \pm 1.84$ colonias $/ \mathrm{m}^{2}$ y $7.58 \pm 2.16$ colonias/ $\mathrm{m}^{2}$. El valor medio más pequeño fue observado en El Mangle, mientras que las mayores cifras fueron reportadas para las localidades de Aguadores este y Aguadores oeste (Cuadro 3).

\section{Agrupamiento de las localidades e índi-} ces ecológicos: $\mathrm{El}$ análisis de agrupamiento basado en la densidad de especies dio lugar a tres grupos de localidades (I-III) (Fig. 1).

CUADRO 2

Composición y porcentaje de abundancia de las especies por localidad y porcentaje total en la Reserva Ecológica Siboney-Juticí

TABLE 2

Composition and percentage of abundance of the species per site and total percentage in the Ecological Reserve Siboney-Juticí

\begin{tabular}{|c|c|c|c|c|c|c|c|}
\hline Especies & $\mathrm{J}^{1}(\%)$ & $\mathrm{M}^{2}(\%)$ & $\mathrm{SE}^{3}(\%)$ & $\mathrm{SO}^{4}(\%)$ & $\operatorname{AE}^{5}(\%)$ & $\mathrm{AO}^{6}(\%)$ & Total $(\%)$ \\
\hline Briareum asbestinum & 8 & - & 3 & 10 & 2 & - & 3 \\
\hline Erythropodium caribaeorum & - & - & - & - & $<1$ & 5 & 1 \\
\hline Muricea muricata & - & - & 1 & - & $<1$ & 5 & 2 \\
\hline Muriceopsis flavida & $<1$ & 20 & 9 & 9 & 4 & 9 & 8 \\
\hline Eunicea calyculata f. typica & - & - & 6 & - & - & 8 & 3 \\
\hline Eunicea mammosa & 5 & 1 & 11 & 10 & $<1$ & 5 & 6 \\
\hline Eunicea succinea f. succinea & - & - & 1 & - & $<1$ & 2 & 1 \\
\hline Eunicea succinea f. plantaginea & 2 & - & 1 & $<1$ & - & $<1$ & 1 \\
\hline Eunicea tourneforti f. tourneforti & 4 & - & 1 & 4 & 4 & 5 & 3 \\
\hline Eunicea flexиosa & 11 & 7 & 33 & 17 & 27 & 15 & 19 \\
\hline Eunicea sp. 1 & - & 13 & - & - & - & - & 1 \\
\hline Eunicea sp. 2 & - & - & - & - & - & $<1$ & $<1$ \\
\hline Plexaura homomalla & 30 & 16 & 7 & 12 & 5 & 5 & 11 \\
\hline Plexaura kuekenthali & $<1$ & - & - & - & - & - & $<1$ \\
\hline Plexaurella dichotoma & 4 & - & 3 & 3 & - & 3 & 2 \\
\hline Plexaurella grisea & - & - & - & $<1$ & - & - & $<1$ \\
\hline Plexaurella nutans & - & - & 2 & - & - & - & $<1$ \\
\hline Pseudoplexaura crucis & - & - & - & - & 2 & - & $<1$ \\
\hline Pseudoplexaura porosa & - & - & - & - & 22 & 5 & 6 \\
\hline Pseudoplexaura flagellosa & 3 & 1 & - & 17 & 1 & - & 3 \\
\hline Gorgonia ventalina & 17 & 29 & 13 & 14 & 14 & 18 & 17 \\
\hline Pseudopterogorgia americana & 16 & 6 & 4 & 3 & 11 & 12 & 9 \\
\hline Pseudopterogorgia elisabethae & - & 7 & - & - & 1 & - & 1 \\
\hline Pterogorgia citrina & - & - & 3 & - & - & - & $<1$ \\
\hline Pterogorgia guadalupensis & - & - & 3 & - & - & - & $<1$ \\
\hline
\end{tabular}

${ }^{1} \mathbf{J}=$ Juticí,${ }^{2} \mathbf{M}=$ El Mangle, ${ }^{3} \mathbf{S E}=$ Sardinero este, ${ }^{4} \mathbf{S O}=$ Sardinero oeste, ${ }^{5} \mathbf{A E}=$ Aguadores este, ${ }^{6} \mathbf{A O}=$ Aguadores oeste. 
CUADRO 3

Densidad, índices de diversidad y severidad y pronóstico ambiental inferido por localidad

TABLE 3

Site density, indexes of diversity and environmental severity and predictability inferred per site

$\begin{array}{lcccl}\text { Localidades } & \begin{array}{c}\left.\text { Densidad (colonias } / \mathrm{m}^{2}\right) \\ (\text { Media } \pm \mathrm{DS})\end{array} & \mathrm{H}^{\prime}{ }^{1} & \mathrm{~J}^{\prime 2} & \text { Estado ambiental inferido } \\ \text { Juticí } & 4.19 \pm 1.5 & 2.04 & 0.821 & \text { Favorable y constante } \\ \text { El Mangle } & 3.58 \pm 1.84 & 1.879 & 0.855 & \text { Severo y constante } \\ \text { Sardinero este } & 4.59 \pm 1.56 & 2.245 & 0.81 & \text { Favorable y constante } \\ \text { Sardinero oeste } & 4.54 \pm 1.64 & 2.216 & 0.892 & \text { Favorable y constante } \\ \text { Aguadores este } & 7.17 \pm 2.46 & 2.116 & 0.781 & \text { Favorable y casi constante } \\ \text { Aguadores oeste } & 7.58 \pm 2.16 & 2.439 & 0.901 & \text { Muy favorable y constante }\end{array}$

${ }^{1} \mathbf{H}$ '=heterogeneidad, ${ }^{2} \mathbf{J}$ '=equitatividad.

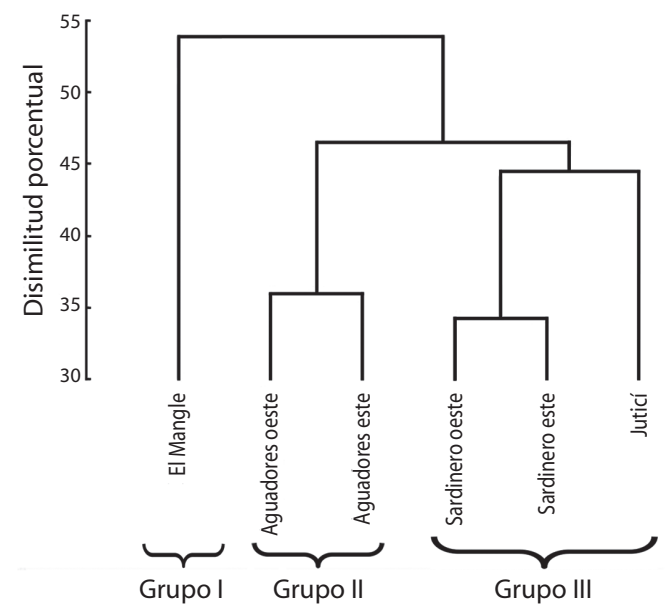

Fig. 1. Agrupación de las localidades de muestreo basado en la densidad de especies utilizando el Índice de Disimilitud de Bray-Curtis.

Fig. 1. Cluster analysis of the sampling sites based on species density using the Bray-Curtis Disimilarity Index.

Grupo I: Constituido solo por El Mangle. Los macizos en esta localidad se caracterizaron por poseer longitudes que oscilaron entre $35 \mathrm{~m}$ y $40 \mathrm{~m}$, con anchos entre $5 \mathrm{~m}$ y $10 \mathrm{~m}$ y alturas de $0.5 \mathrm{~m}$ a $1.5 \mathrm{~m}$. El relieve presentó abundantes, aunque poco profundos, desniveles y oquedades, cubiertos por cieno y una gruesa capa de arena. Aquí se reportaron las densidades más bajas, siendo G. ventalina, P. homomalla y $M$. flavida, las especies más abundantes. Los índices de H' y J' reflejaron condiciones ambientales severas y constantes (Cuadro 3). El ITH (37\%) fue bajo y el ICC (9\%) fue el más bajo calculado en el área de estudio (Cuadro 4).

Grupo II: Las dimensiones de los macizos de este grupo oscilaron entre $50 \mathrm{~m}$ y $80 \mathrm{~m}$ de longitud, $20 \mathrm{~m}$ y $40 \mathrm{~m}$ de ancho y una altura superior a los $2 \mathrm{~m}$, con relieves relativamente uniformes y sometidos a la sedimentación. En este grupo la densidad fue moderada, siendo E. flexuosa, Pseudoplexaura porosa (Houtuyn 1772), G. ventalina y P. americana, las especies más abundantes. A partir de los índices de

\section{CUADRO 4}

Índice de Tensión Hidrodinámica (ITH) e Índice Comparativo de Contaminación (ICC) por localidad

TABLE 4

Hydrodynamic Stress Index (ITH) and Comparative Pollution Index (ICC) per site

$\begin{array}{lcc}\text { Localidades } & \text { ITH }(\%) & \text { ICC (\%) } \\ \text { Juticí } & 39 & 23 \\ \text { El Mangle } & 37 & 9 \\ \text { Sardinero este } & 73 & 51 \\ \text { Sardinero oeste } & 47 & 48 \\ \text { Aguadores este } & 51 & 38 \\ \text { Aguadores oeste } & 60 & 33\end{array}$


H' y J', se infirió un estado ambiental favorable y casi constante para la localidad Aguadores este y muy favorable y constante para Aguadores oeste (Cuadro 3). El ITH mostró valores moderados con tendencia a ser altos (51-60\%) y el ICC reflejó una tendencia a ser moderado (33-38\%) (Cuadro 4).

Grupo III: Reunió las localidades con macizos de $25 \mathrm{~m}-50 \mathrm{~m}$ de longitud, hasta $20 \mathrm{~m}$ de ancho y entre $1.5 \mathrm{~m}$ y $2 \mathrm{~m}$ de alto. Presentaban relieves poco accidentados, con algunas oquedades y desniveles cubiertos por arena y cieno. En este grupo las densidades fueron bajas y las especies E.flexuosa, G. ventalina y $P$. homomalla, fueron las más abundantes. Los índices de H'y J' evidenciaron ambientes favorables y constantes (Cuadro 3 ). Los valores de ITH oscilaron entre bajos y altos (39-73\%) y los del ICC entre bajos y moderados (23-51\%) (Cuadro 4). De manera general los índices de H' y J' fueron relativamente altos, por lo que el tipo de ambiente inferido más frecuente fue favorable y constante (Cuadro 3).

Colonias afectadas: Del total de colonias vivas contadas, el $13 \%$ presentó algún tipo de afectación, siendo el deterioro por daños mecánicos la causa principal (4\% del total de colonias contadas) (Cuadro 5). No se observaron individuos de $H$. carunculata, tampoco se presenció blanqueamiento ni las enfermedades de Banda Roja y Banda Negra.

La especie de macroalga más común sobre los octocorales fue Dictyota pulchela Hörnig \& Schnetter 1988; se identificó también Lobophora variegata (Lamouroux) Womersley ex Oliveira 1977. P. homomalla y P. americana constituyeron las especies más afectadas provocadas por el molusco $C$. gibbosum. Se observó un ejemplar de Cyphoma signatum Pilsbry \& McGinty 1939 sobre una colonia de P. dichoto$m a$, en la localidad de Aguadores oeste. El resto de los invertebrados observados fueron bivalvos e hidrozoos. La especie que más colonias afectadas presentó en sus poblaciones fue $G$. ventalina $(34 \%)$, con daños mecánicos, pérdida de cenénquima, sobrecrecimiento de macroalgas y de M. alcicornis u otros invertebrados, depredación por $C$. gibbosum y crecimiento de tumores algales.

El número de colonias muertas fue bajo (36 colonias) (Cuadro 5). La mortalidad estuvo

\section{CUADRO 5}

Número de colonias muertas, porcentaje de colonias sanas y afectadas por localidad y total para la Reserva Ecológica Siboney-Juticí y tipo de daño observado

TABLE 5

Number of dead colonies, percentage of healthy and injured colonies per site and total in the Ecological Reserve Siboney-Jutici, and type of injury observed

\begin{tabular}{|c|c|c|c|c|c|c|c|}
\hline \multirow{2}{*}{ Afectaciones } & \multicolumn{6}{|c|}{ Localidades de muestreo } & \multirow{2}{*}{ Total } \\
\hline & $\mathrm{J}^{1}$ & $\mathrm{M}^{2}$ & $\mathrm{SE}^{3}$ & $\mathrm{SO}^{4}$ & $\mathrm{AE}^{5}$ & $\mathrm{AO}^{6}$ & \\
\hline No. colonias muertas & 4 & 7 & 6 & 5 & 6 & 8 & 36 \\
\hline Sanas & 88 & 80 & 85 & 93 & 83 & 91 & 87 \\
\hline Afectadas & 12 & 20 & 15 & 7 & 17 & 9 & 13 \\
\hline Daños mecánicos & 5 & 12 & 1 & 1 & 9 & $<1$ & 4 \\
\hline Pérdida de cenénquima & 5 & - & 5 & 2 & 6 & 1 & 3 \\
\hline Presencia de $C$. gibbosum & 3 & 5 & 2 & 3 & 2 & 2 & 3 \\
\hline Presencia de $M$. alcicornis & - & 4 & 6 & 3 & $<1$ & 3 & 2 \\
\hline Presencia de macroalgas & - & - & 4 & 3 & 6 & 2 & 3 \\
\hline Presencia de otros invertebrados & 1 & - & - & - & $<1$ & $<1$ & $<1$ \\
\hline Aspergilosis & - & - & - & - & - & $<1$ & $<1$ \\
\hline Tumores algales & - & 1 & - & - & - & - & $<1$ \\
\hline
\end{tabular}

${ }^{1} \mathbf{J}=$ Juticí, ${ }^{2} \mathbf{M}=$ El Mangle, ${ }^{3} \mathbf{S E}=$ Sardinero este, ${ }^{4} \mathbf{S O}=$ Sardinero oeste, ${ }^{5} \mathbf{A E}=$ Aguadores este, ${ }^{6} \mathbf{A O}=$ Aguadores oeste. 
dada por el sobrecrecimiento de M. alcicornis y probablemente por el desprendimiento del sustrato. En el mangle se reportó la mayor proporción entre colonias muertas y colonias vivas y el mayor porcentaje de colonias afectadas (Cuadro 5).

\section{DISCUSIÓN}

Composición y abundancia: En la Reserva Ecológica Siboney-Juticí se identificó el $32 \%$ de las 77 especies de alcionáceos reportadas para Cuba (Hernández-Muñoz \& González-Ferrer 2007, Varela et al. 2008). La familia Plexauridae fue la mejor representada, lo que coincide con Guzmán \& Cortés (1985), quienes plantearon que este constituía un patrón de diversidad frecuente en el Caribe. No obstante, el número de especies de esta familia fue menor que el reportado por Alcolado (1981) en la costa sur de Granma y Santiago de Cuba y por Chiappone et al. (2001) en la terraza de la Base Naval de la Bahía de Guantánamo, debido probablemente al amplio rango batimétrico y espacial que abarcaron estas investigaciones.

Agrupamiento de las localidades, densidad e índices ecológicos: En el grupo I, las bajas densidades pudieron deberse a la baja altura de los macizos y la pequeña distancia existente entre ellos, lo que puede propiciar que en los canales se concentren las corrientes, aumentando su velocidad y provocando la resuspensión de los sedimentos depositados en ellos. Esto limita el desarrollo de colonias de otras especies de octocorales, fundamentalmente en las zonas bajas, siendo la causa de que se infirieran condiciones severas pero constantes. La tensión hidrodinámica determinada en este grupo corresponde con la encontrada por Alcolado (1981), quien reportó que para la región sur oriental de Cuba, entre $10 \mathrm{~m}$ y $15 \mathrm{~m}$ de profundidad, ocurría una inversión de los porcentajes de especies resistentes y poco resistentes a la turbulencia ("profundidad de inversión").

En el grupo II se observaron los valores más altos de densidad de la región. La alta diversidad observada en este grupo coincide con las observaciones de Guardia \& González-Sansón (2000), quienes encontraron una relación directa entre la diversidad, la densidad de octocorales, la sedimentación y la eutrofización. Esto contradice lo planteado por Herrera-Moreno (1991), quien señaló que una de las causas de la disminución de la diversidad era la influencia de ríos. Se esperaba que los estados ambientales inferidos para cada localidad de este grupo, ambiente casi contante en Aguadores este y constante en Aguadores oeste, estuvieran invertidos, debido a que estas localidades están ubicadas en los lados que sus nombres indican en la desembocadura del río San Juan y las corrientes marinas en la región sur oriental de Cuba se mueven de este a oeste (Arrianza et al.2008). Es probable que la causa principal radique en la distancia a la desembocadura del río, pues la localidad Aguadores oeste se encuentra aproximadamente a una distancia dos veces mayor que la de Aguadores este. Por consiguiente esta última localidad puede depender más de las fluctuaciones en los aportes que el río hace al medio marino que Aguadores oeste.

La incidencia de la tensión hidrodinámica sobre el grupo II puede estar relacionada con la uniformidad del relieve de los macizos, lo que provoca que las corrientes y el batimiento por el oleaje incidan directamente sobre la distribución de los octocorales, al no encontrar obstáculos considerables que cambien su dirección e intensidad, lo que corresponde con conclusiones hechas por Yoshioka \& Yoshioka (1989) en algunos arrecifes de Puerto Rico. En este ecosistema solo la profundidad pudo ejercer un efecto amortiguador.

En el grupo III, a pesar que el relieve presentó pocas irregularidades, la proporción entre el número de depresiones y el tamaño relativamente pequeño de algunos macizos pudo limitar la colonización de un mayor número octocorales, debido a la escasez de sustrato libre de sedimentos, razón de las bajas densidades observadas. En este grupo se destacó la estabilidad poblacional de la especie $P$. homomalla; especie con resistencia a la 
sedimentación, que predominó en las depresiones en el relieve de los macizos, lo que coincide con lo reportado por Kinzie (1973), quien la observó en la zona de laguna del arrecife y en canales donde se depositaban abundantes sedimentos. La abundancia de G. ventalina, E. flexuosa y E. mammosa, consideradas por Alcolado (1981) como sensibles a la sedimentación, estuvo relacionada con elevaciones en los sustratos rocosos; lo que coincide con lo observado por Herrera-Moreno \& Alcolado (1986) en arrecifes de Santa Cruz del Norte. De manera general, estos resultados corroboran lo planteado por Bayer (1961), Torruco \& González (2000) y Hernández-Fernández (2004), acerca del papel determinante que posee la disponibilidad y naturaleza del sustrato en la distribución de los octocorales.

A pesar que los valores de ITH en este grupo son los esperados para la profundidad a la que se realizó el estudio, es probable que se sobrestimara en Sardinero este, debido a que la mayoría de las especies resistentes a la turbulencia observadas en esta localidad también lo son a la sedimentación (i.e. E. calyculata f. calyculata, E. tourneforti, M. muricata, $P$. dichotoma, P. citrina y P. guadalupensis). Este factor pudo tener cierta incidencia debida a la presencia de canales de alrededor de $50 \mathrm{~m}$ de amplitud.

Algo similar pudo suceder con la contaminación ya que aparentemente no existen fuentes contaminantes en la zona. Por otro lado, sería erróneo asociar a P. flagellosa, especie abundante en Sardinero oeste, con algún ambiente en particular, debido a la variedad de criterios en torno a los hábitats en los que comúnmente se desarrolla. Esta especie fue reportada por Kinzie (1973) como moderadamente abundante entre $15 \mathrm{~m}$ y $30 \mathrm{~m}$ en cabezos del arrecife ubicados dentro de canales con arena. Fue considerada además por Alcolado (1981) como moderadamente resistente a la turbulencia y la encontró entre $2 \mathrm{~m}$ y $30 \mathrm{~m}$ y en abundancia tanto en zonas traseras como de embate en algunos arrecifes. A su vez, Herrera-Moreno \& Alcolado (1986) reportaron poblaciones de mediano y pequeño tamaño en casi todos los perfiles estudiados en $5 \mathrm{~m}, 10 \mathrm{~m}$ y $15 \mathrm{~m}$ de profundidad, donde la turbulencia inferida varió de muy alta a baja. Recientemente, fue observada con escasa abundancia en Bahía de Cochinos (Varona et al. 2004), en perfiles con bajo ITH; y en el 2005 , estos mismos autores, la reportaron en arrecifes ubicados en la región norte oriental de La Habana, con valores de ITH que fluctuaron entre bajos y muy altos.

Los valores generales de densidad observados en la Reserva Ecológica Siboney-Juticí fueron similares a los encontrados por Hernández-Fernández (2004) (5.31-5.87 colonias/ $\mathrm{m}^{2}$ ) en el hábitat de macizos y canales del Área Protegida Centro-Oeste Cayo Coco, zona norte central de Cuba. Sin embargo, tendieron a ser bajos si se compara con los máximos reportados por Yoshioka (1997) de 62 colonias $/ \mathrm{m}^{2}$ para Puerto Rico y 20 colonias $/ \mathrm{m}^{2}$ para Cuba (Alcolado 2009, com. pers.).

Colonias afectadas: Durante el período de tiempo que abarcó el muestreo la comunidad de octocorales mostró un buen estado de conservación. Los daños mecánicos fueron causados probablemente por la acción del oleaje y las corrientes, incrementándose cuando actúan en sinergia con la aspergilosis y el sobrecrecimiento de macroalgas, los cuales pueden debilitar la estructura de la colonia. Fue notable su incidencia en El Mangle, debido tal vez a la abundancia de G. ventalina, especie susceptible a sufrir este tipo de daño. El alto número de colonias afectadas en $G$. ventalina pudo deberse, en parte, a su forma de crecimiento, ya que abarca superficies muy amplias, ofreciendo una alta resistencia al movimiento del agua, lo que la hace susceptible a dañarse por los corales pétreos y por los objetos que se mueven en la columna de agua y sobre el fondo.

La pérdida de cenénquima pudo ser consecuencia fundamentalmente del efecto abrasivo de la arena. Se descarta la posibilidad de que el roce entre las colonias haya sido una de las causas en Juticí y Sardinero este, debido a las bajas densidades observadas; no obstante, es probable que en Aguadores oeste dicho factor haya actuado en sinergia con la abrasión 
por la arena, como observó Wahle (1985) en algunos arrecifes de Jamaica. Las especies con mayores daños provocados por $C$. gibbosum no coinciden con las observaciones hechas por Behety (1980), Harvell \& Suchanek (1987) y Botero (1990) en diversas regiones del Caribe. Es notable el escaso número de colonias con tumores algales, solo una de G. ventalina, si se compara con las observaciones de Goldberg et al. (1984), quienes reportaron una alta incidencia sobre colonias de G. ventalina y Pseudoplexaura spp., y Botero (1990), quien observó tumores además en E.flexuosa, aunque no en G. ventalina.

Las causas de muerte de octocorales identificadas, desprendimiento del sustrato y sobrecrecimento de M. alcicornis, coinciden con las observadas por Wahle (1985) en arrecifes de Jamaica. Varona et al. (2004, 2005) también las reportaron como responsables de la muerte de los octocorales en sus respectivos estudios, aunque en ninguno de los casos superó el $1 \%$. El mayor número de colonias muertas con respecto al total contabilizado en una localidad del Mangle, pudo ser consecuencia de la combinación del batimiento y la presencia de gran cantidad de arena sobre los macizos, que constituyeron sustratos no consolidados para su asentamiento, lo que concuerda con lo expuesto por Bayer (1961) y Kinzie (1973).

Sin embargo se hace necesario implementar un conjunto de acciones encaminadas a garantizar la conservación del hábitat en el que se desarrollan las comunidades de octocorales como: incorporar al plan de manejo, acciones que generen una relación comunidad-reserva estimulando el turismo ecológico y acciones de educación ambiental, programas de divulgación para dar a conocer la reserva, sus valores, importancia y vías de uso sostenible de los recursos naturales en particular los marinos; hacer énfasis en las problemáticas de pesca, recolección y contaminación. Además realizar limpieza periódica de la línea de costa y sector hasta los $20 \mathrm{~m}$ de profundidad, incorporar a las comunidades cercanas mediante programas de educación ambiental. Se prestará especial atención a desechos plásticos y restos de artes de pesca en arrecifes y cabezos. Es importante fortalecer la actividad de vigilancia por parte de las entidades reguladoras y la implementación de la legislación ambiental vigente. Evaluar en diferentes condiciones los aportes de sedimentos y su dispersión en las cuencas de los ríos San Juan, Sardinero, Juticí y Carpintero. Particularizar en la dinámica de las cuencas de Juticí y Sardinero. Priorizando acciones de reforestación en las cuencas de los Ríos Sardinero y San Juan para mitigar estos impactos.

También es necesario ubicar puntos de monitoreo de biodiversidad marina por hábitats y la creación de un programa de investigaciones que aborde aspectos particulares como: dinámica de los daños sobre las poblaciones de $G$. ventalina y efecto de la ocurrencia de eventos extremos (e.g. ciclones tropicales, temperaturas excesivamente altas, derrames de contaminantes y epidemias) para conocer respuestas y adaptabilidad de las comunidades de octocorales.

En la Reserva Ecológica Siboney-Juticí se encontraron ambientes favorables y constantes para el desarrollo de los octocorales y poblaciones con densidades que tendieron a ser bajas, siendo E. flexuosa y G. ventalina las especies más abundantes. Las localidades de muestreo, en sentido general, mostraron similitudes en dependencia de la cantidad de sustrato disponible para el asentamiento de las plánulas de los octocorales y sus poblaciones evidenciaron un buen estado de conservación, si se tiene en cuenta la proporción entre colonias dañadas y sanas. La mitigación (hasta donde sea posible) de las fuentes de perturbación a través de planes de manejo a corto, mediano y largo plazo; garantizará que las comunidades de octocorales de la Reserva Ecológica Siboney-Juticí mantengan su composición, estructura y función.

\section{AGRADECIMIENTOS}

Agradecemos a Leopoldo Viña (Polo), José Tamaño (Yiyo), Asdrúbal Viña, Yoendrys Paz y Luis Reyes por su colaboración insustituible en el trabajo de campo. A Pedro M. Alcolado, Alejandro Herrera Moreno y Elena 
de la Guardia y a los revisores anónimos por sus contribuciones y oportunos comentarios. Al Departamento de Biología de la Universidad de Oriente, al Centro Oriental de Ecosistemas y Biodiversidad (BIOECO) del Ministerio de Ciencia, Tecnología y Medio Ambiente (CITMA) por haber propiciado y apoyado esta investigación y al Centro de Investigaciones de Ecosistemas Costeros (CIEC) del CITMA por su apoyo y recomendaciones.

\section{RESUMEN}

Se estudió la comunidad de octocorales del arrecife de macizos y canales de la Reserva Ecológica SiboneyJuticí (sur oriente de Cuba) con el objetivo de caracterizar su composición, estructura y estado de conservación, así como inferir a partir de estas las condiciones ambientales imperantes en el área. La investigación se realizó entre enero y junio de 2009, en seis localidades ubicadas entre $12 \mathrm{~m}$ y $17 \mathrm{~m}$ de profundidad. Como unidad de muestreo se empleó un marco de $1 \mathrm{~m}^{2}$, el cual fue puesto en zigzag cada $2 \mathrm{~m}$ sobre la superficie del arrecife de macizos. Se determinó, por localidad, la densidad de colonias y se infirieron los grados de severidad, constancia ambiental, tensión hidrodinámica y el Índice Comparativo de Contaminación. Se determinaron, además, los daños en la comunidad de octocorales. Se analizaron 752 colonias e identificaron 25 especies de octocorales. Eunicea flexuosa y Gorgonia ventalina fueron las especies más abundantes. La densidad fluctuó entre $3.58 \pm 1.84$ colonias $/ \mathrm{m}^{2}$ y $7.58 \pm 2.16$ colonias/ $\mathrm{m}^{2}$ (densidad de baja a moderada tendiendo a baja). En la zona se infirió una tensión hidrodinámica entre baja y alta, con niveles de contaminación bajos y moderados, y un ambiente generalmente favorable y constante. Los daños de tipo mecánico fueron los más frecuentes, siendo $G$. ventalina la especie con más colonias dañadas. La comunidad de octocorales del hábitat de macizos y canales de la Reserva Ecológica Siboney-Juticí presentó un buen estado de conservación.

Palabras clave: octocorales, arrecife de macizos y canales, composición, estructura, daños, Cuba.

\section{REFERENCIAS}

Alcolado, P.M. 1981. Zonación de los gorgonáceos someros de Cuba y su posible uso como indicadores comparativos de tensión hidrodinámica sobre organismos del bentos. Inf. Cient. Téc. Inst. Oceanol. 187: 1-43.
Alcolado, P.M. 1992. Sobre la interpretación del medio marino mediante el empleo de los índices de diversidad y equitatividad. Cien. Biol. 24: 124-127.

Arrianza, L., J. Simanca, L. Rodas, S. Lorenzo, M. Hernández, E. Linares, D. Milan \& P. Romero. 2008. Corrientes marinas estimadas en la plataforma suroriental cubana. Ser. Oceanol. 4: 1-10.

Bayer, F.M. 1961. The shallow-water Octocorallia of the West Indian region. A manual for marine biologists. Martinus Nijhoff, La Haya, Holanda.

Behety, P.A. 1980. Variaciones en la dieta de Cyphoma gibbosum Linnaeus en función de la profundidad. Cien. Biol. 5: 75-84.

Botero, L. 1990. Obsevations on the size predators and tumor-like outgrowths of gorgonian octocoral colonies in the area of Santa Marta, Caribbean Coast of Colombia. NE Gulf Sci. 11: 1-10.

Bray, R.J. \& J.T. Curtis. 1957. An ordination of the upland forest communities of southern Wisconsin Ecol. Monogr. 27: 325-349.

Chiappone, M., K. Sullivan-Sealey, G. Bustamante \& J. Tschirky. 2001. A rapid assessment of coral reef community structure and diversity patterns at naval station Guantanamo Bay, Cuba. Bull. Mar. Sci. 69: 373-394.

García-Parrado, P. \& P.M. Alcolado. 1996. Catálogo de los Octocorales (Cnidaria) de Cuba, con comentarios sobre su taxonomía. Avicennia 4/5: 41-45.

Goldberg, W.M. 1973. The ecology of the coral-octocoral communities off the southeast Florida coast: geomorphology, species composition, and zonation. Bull. Mar. Sci. 23: 465-488.

Goldberg, W.M., J.C. Makenson \& S.B. Colley. 1984. Entocladia endozoica sp. nov., a pathogenic chorophyte: structure, life history, physiology, and effect on its coral host. Biol. Bull. 166: 368-383.

González-Díaz, P., E. de la Guardia \& G. GonzálezSansón. 2003. Efecto de los efluentes terrestres sobre las comunidades bentónicas de arrecifes coralinos de Ciudad de La Habana, Cuba. Rev. Invest. Mar. 24: 193-204.

González-Ontivero, O., D. Macías \& E. de la Guardia. 2007. Evaluación de los corales escleractíneos y gorgonias en dos localidades de Ciudad de La Habana, Cuba. Rev. Invest. Mar. 28: 21-27.

de la Guardia, E. \& G. González-Sansón. 2000. Asociaciones de corales, gorgonias y esponjas en el sublitoral 
habanero al oeste de la Bahía de La Habana, II. Índices ecológicos. Rev. Invest. Mar. 21: 9-26.

Guitart-Manday, D. 1959. Gorgonias del litoral de la costa norte de Cuba. Ser. Téc. Acuario Nac. 1: 1-24.

Guzmán, H.M. \& J. Cortés. 1985. Organismos de los arrecifes coralinos de Costa Rica. IV: Descripción y contribución geográfica de octocoralios (Cnidaria: Anthozoa) de la costa Caribe. Brenesia 24: 125-173.

Harvell, C.D. \& T. Suchanek. 1987. Partial predation on tropical gorgonians by Cyphoma gibbosum (Gastropoda). Mar. Ecol. Prog. 38: 37-44.

Hernández-Fernández, L. \& P.M. Alcolado. 2007. Estructura de la comunidad de octocorales de una zona propuesta como área protegida en Cayo Coco, Cuba. Rev. Invest. Mar. 28: 209-216.

Hernández-Fernández, L. 2004. Composición de las comunidades de octocorales del Área Protegida Centro-Oeste Cayo Coco, Cuba. Tesis de maestría, Universidad de La Habana, La Habana, Cuba.

Hernández-Muñoz, D. \& S. González-Ferrer. 2007. Gorgonias - Filo CNIDARIA, clase ANTHOZOA, subclase OCTOCORALLIA. Lista de especies registradas en Cuba. In R. Claro (ed.). La Biodiversidad marina de Cuba (CD-ROM). Instituto de Oceanología, La Habana, Cuba.

Herrera-Moreno, A. \& P.M. Alcolado. 1983. Efectos de la contaminación sobre las comunidades de gorgonáceos al Oeste de la Bahía de La Habana. Cien. Biol. 10: 69-85.

Herrera-Moreno, A. \& P.M. Alcolado. 1986. Estructura ecológica de las comunidades de gorgonáceos del arrecife de Santa Cruz del Norte. Rep. Invest. Inst. Oceanol. 49: 1-27.

Herrera-Moreno, A. 1991. Efectos de la contaminación sobre la estructura ecológica de los arrecifes coralinos en el litoral habanero. Tesis de doctorado, Instituto de Oceanología, La Habana, Cuba.

Kinzie, R.A. 1973. The zonation of West Indian gorgonians. Bull. Mar. Sci. 23: 93-155.
Ludwing, J.A. \& J.F. Reynolds. 1988. Statistical Ecology: A primer on methods and computing. Wiley, Nueva York, EEUU.

Pielou, E.C. 1966. The measurement of diversity in different types of biological collections. J. Theor. Biol. 13: 131-144.

Preston, E.C. \& L. Preston. 1975. Ecological structure in a West Indian gorgonian fauna. Bull. Mar. Sci. 25: 248-254.

Shannon, C.E. \& W. Weaver. 1949. The mathematical theory of communication. Urbana Univ. Illinois, EEUU.

Torruco, D. \& M.A. González. 2000. Distribución de los octocorales del Majahual, costa de Quintana Roo, México. Avicennia 12/13: 1-10.

Varela, C., M.V. Orozco \& G. Varona. 2008. Registros nuevos de octocorales (Cnidaria: Anthozoa: Octocorallia) para Cuba. Cocuyo 17: 10-11.

Varona, G., H. Caballero \& E. de la Guardia. 2004. Estructura ecológica de las comunidades de octocorales en la costa oriental de Bahía de Cochinos, Cuba. Rev. Invest. Mar. 25: 209-218.

Varona, G., H. Caballero \& E. de la Guardia. 2005. Estructura ecológica de las comunidades de gorgonáceos de la costa norte oriental de La Habana. Rev. Invest. Mar. 26: 27-36.

Wahle, C.M. 1985. Habitat-related patterns of injury and mortality among Jamaican gorgonians. Bull. Mar. Sci. 37: 905-927.

Weinberg, S. 1981. A comparison of Coral Reef Survey Methods. Contrib. Zool. 51: 199-218.

Yoshioka, P. 1997. Are variations in gorgonian recruitment determined by "pre-settlement" or "post-settlement" processes? Proceedings of the $8^{\text {th }}$ International Coral Reef Symposium II: 1175-1178.

Yoshioka, P.M. \& B.B. Yoshioka. 1989. Effects of wave energy, topographic relief and sediment transport on the distribution of shallow-water gorgonians of Puerto Rico. Coral Reefs 8: 145-152. 